Original article

\title{
Understanding variations in the use of hypofractionated radiotherapy and its specific indications for breast cancer: A mixed-methods study
}

\author{
Joan Prades a , Manel Algara ${ }^{c}$, Josep A. Espinàs ${ }^{a}$, Blanca Farrús ${ }^{\mathrm{d}}$, Meritxell Arenas ${ }^{\mathrm{e}, \mathrm{f}}$, Victoria Reyes ${ }^{\mathrm{g}}$, \\ Virginia García-Reglero $^{\mathrm{h}}$, Maria Josep Cambra ${ }^{\mathrm{i}}$, Esther Rubio ${ }^{\mathrm{j}}$, Lluis Anglada ${ }^{\mathrm{k}}$, Arantxa Eraso ${ }^{\mathrm{b}, \mathrm{l}}$, \\ Agustí Pedro $^{\mathrm{m}}$, Maria J. Fuentes-Raspall ${ }^{\mathrm{n}}$, Victòria Tuset ${ }^{\mathrm{o}}$, Judit Solà ${ }^{\mathrm{a}}$, Josep M. Borras ${ }^{\mathrm{a}, \mathrm{b}, *}$

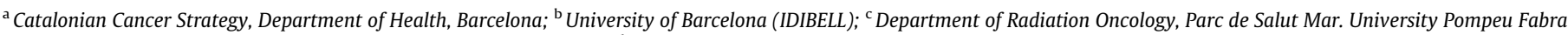

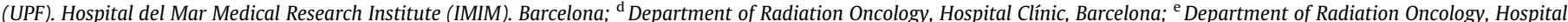

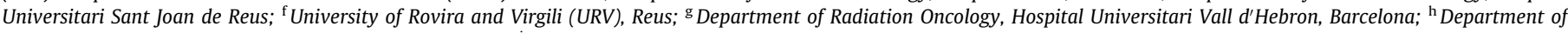

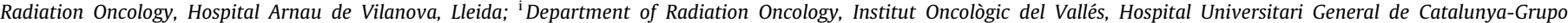

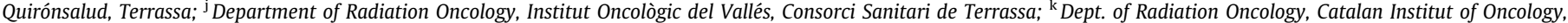

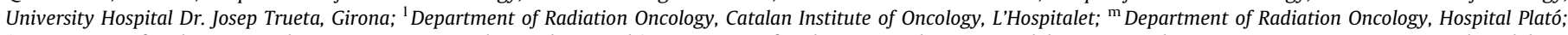

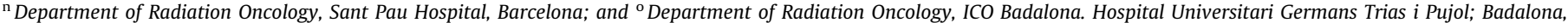 \\ Spain
}

\section{A R T I C L E I N F O}

\section{Article history:}

Received 4 November 2016

Received in revised form 23 January 2017

Accepted 25 January 2017

Available online $\mathrm{xxxx}$

\section{Keywords:}

Dose hypofractionation

Breast neoplasms

Mixed-methods

Radiation oncology

\begin{abstract}
A B S T R A C T
Background and purpose: Radiation oncology guidelines favour hypofractionated whole-breast radiotherapy (HWBRT) over more conventional schemes in the conservative treatment of breast cancer, but its adoption still varies in clinical practice. This study assessed the patterns of HWBRT adoption in Catalonia (Spain).

Material and methods: We used a mixed-methods approach based on an explanatory sequential design, first collecting and analysing quantitative data on HWBRT use ( $>2.5$ Gy per fraction) in 11 public radiotherapy centres (2005-2015) and then performing 25 semi-structured interviews with all department heads and reference radiation oncologist/s.

Results: Of the 34,859 patients fulfiling the study criteria over the study period, just $12 \%$ were hypofractionated, reaching a percentage of $29 \%$ in $2015(p<0.001)$. Our analysis showed a narrowing age gap between patients receiving conventional fractionation and hypofractionation in centres leading adoption. However, there were important differences in clinicians' interpretation of evidence (e.g. regarding the perceived risk of long-term toxicity) and selection of patients for specific indications, both within and between departments.

Conclusions: Differences observed in the rate of adoption of HWBRT could not be tackled only using a rational, evidence-based approach. Factors related to the management of radiotherapy departments play a major role in the diffusion of therapeutic strategies.
\end{abstract}

(c) 2017 Elsevier B.V. All rights reserved. Radiotherapy and Oncology xxx (2017) xxx-xxx
A consolidated body of evidence [1-4] has shown that a high proportion of breast cancer patients may achieve the same clinical outcomes with 15-16 fractions (40-42.5 Gy) of postoperative radiotherapy as with the longer conventional radiotherapy course of 25 fractions. Randomized studies show that this conservative regimen is more convenient for patients, incurs a lower cost for the healthcare system, and causes less acute skin toxicity [5]. From a cancer care policy standpoint, the American Society for Radiation Oncology (ASTRO), the European Society for Radiotherapy \&

* Corresponding author at: University of Barcelona, IDIBELL. Hospital Duran i Reynals; Gran via 199; 08908-Hospitalet, Barcelona, Spain.

E-mail address: jmborras@iconcologia.net (J.M. Borras).
Oncology (ESTRO), and the National Institute for Health and Care Excellence (NICE) all prefer the so-called modest hypofractionation (2-3 Gy per fraction) [6] for most patients with early breast cancer, recognizing the positive implications for health systems with high caseloads of patients potentially undergoing such regimen. Indeed, hypofractionation schedules reduce acute toxicity, which can lead to discontinuation of radiotherapy treatment [7]. Despite the benefits for patients and health systems and its consideration as a standard of care [8,9], hypofractionated whole-breast radiotherapy (HWBRT) still encounters resistance, and its adoption varies in clinical practice $[10,11]$.

Although other strategies-such as breast-intensity modulated radiotherapy (IMRT)-have been adopted on the basis of less 
evidence and higher cost [12], some authors argue that randomized evidence and published guidelines alone are not sufficient grounds to adopt HWBRT [10]. Past discussions regarding tumour-grade sensitivity to hypofractionation [13,14], the unclear effect of biological sub-types in the efficacy of the hypofractionated schedule [8], or breast reconstruction [15] might be contributing to the slow path of adoption and limited utilisation. Caution is still warranted in patients under 40 and in those receiving primary systemic treatment [16] or regional node irradiation (RNI) [17]. Thus, particularly in a context in which the regional clinical guideline does not specifically address this issue [18], departments may interpret evidence for specific indications and nonclinical factors differently, which would explain the existing variability in its adoption.

Our study aims to provide an overview of the adoption of hypofractionation for breast cancer in the Public Health Service of Catalonia (Spain). We combine quantitative and qualitative approaches to evaluate the evolution of its use from 2005 to 2015 and to explain the data from the perspective of radiation oncologists involved in breast cancer treatment in the region.

\section{Materials and methods}

We used a mixed-methods approach based on an explanatory sequential design, which consists of two different interactive phases $[19,20]$. First we collected quantitative data on hypofractionation use; in light of the wide variation of our results, we added a qualitative study in radiotherapy departments. Exploring participants' views in depth helped to explain statistical results by both disentangling the specific indications described at each department and the clinical rationale behind them [21]. Our final interpretation and analysis considered the interaction between quantitative and qualitative findings [22]. The reasons for mixing quantitative and qualitative methods were completeness for a more comprehensive account of the area of inquiry and discovery of hypotheses [19].

We assessed the use of hypofractionation for the 11 public radiotherapy centres in Catalonia (Spain), which provide oncology treatments for a population of 7.5 million and comprise a total of 35 linear accelerators. The longest distance between the home of an individual requiring radiation treatment in Catalonia and a facility is $170 \mathrm{~km}$, while $80 \%$ of the population lives within $20 \mathrm{~km}$. The cost of treatments is reimbursed on the basis of four levels of complexity, regardless of the fractions used.

\section{Quantitative assessment}

We assessed the use of hypofractionation for patients receiving breast cancer treatment with a curative intent in 2005-2015, using data from the Catalonian Hospital Reimbursement Database, which includes all patients receiving a course of radiotherapy. It collects data on sex, age, radiotherapy centre, aim of treatment, tumour site, total dose, planning system, initiation and finalisation of treatment, and number of sessions. It is mandatory to fill out for reimbursement. The criterion for radiotherapy to be considered hypofractionated was $>2.5 \mathrm{~Gy}$ per fraction. The doses included in this study ranged from 2.67 to $3.00 \mathrm{~Gy}$. We assessed differences in patterns of use with descriptive statistics and logistic regression, using SPSS (version 21.0, 2012) and STATA (version 12) software.

\section{Qualitative assessment}

The qualitative study consisted of 25 semi-structured on-site interviews held in October-December 2015 with all department heads and reference breast cancer radiation oncologist/s at each hospital. One-on-one interviews ensured that all critical points were addressed, and the 45-60 min sessions were flexible enough to enable participants to volunteer information on topics relevant to them. Only at the end of each interview was provided anonymised information about the centre's HWBRT utilisation relative to other centres (Figs. 1 and 3). The evaluation of HWBRT, with no comparative information, allowed us to limit the risk of information bias and to contribute to the internal validity of the study based on strictly local perspectives from each service. All interviews were audio-taped and transcribed [23]. These data were then compiled into a documentary record and rendered anonymous.

To analyse the data, we applied thematic-analysis criteria, which emphasise the meaning of the text and interpret its thematic content $[24,25]$. After checking saturation of information [26], we read through to identify general themes and thematic categories to ensure interpreter consensus. We compared interviews to capture recurring views and related experiences [27]. A systematic process of data-treatment analysis was facilitated by the use of the Atlas-ti 6.2 software [28]. Coding and interpretation consistency was checked during analysis by reviewing the transcripts at different moments in time.

\section{Results}

\section{Quantitative assessment}

Of the 34,859 patients fulfiling the study criteria, only 4,322 (12.4\%) breast cancer patients received hypofractionation in the 11 public radiotherapy departments in Catalonia in 2005-2015, with important variations in use across departments $(p<0.001)$. In $2015,29 \%$ of patients received hypofractionated radiotherapy (table 1$)$. While the scheme $(2.67-3.0 \mathrm{~Gy})$ ranged from $1 \%$ to $36.6 \%$ in 2010 among different departments, these figures rose to $8.9 \%$ and $74.7 \%$ in 2015 (Fig. 1). The use of hypofractionation in 2015 exceeded $50 \%$ in two departments, while another five used the therapy $25-50 \%$ of the time, and four others less than $25 \%$ of the time.

Likewise, the median age of patients in 2015 appeared relevant when deciding the regimen to be applied; the more hypofractionation is used, the smaller the age difference between patients who receive it and those who don't (Fig. 2). By contrast, patients' age was near or over 70 years in the four departments with lower rates of HWBRT use.

The trends in the adoption of hypofractionation varied significantly by department, and in several individual cases we observed a striking, non-linear behaviour (Fig. 3). Three milestones in this time period frame the observed variability from a health system perspective. First, three centres (1, 2 and 9 ) introduced hypofractionation in routine practice in 2008 and 2011, in one case it had completely superseded the conventional scheme by 2015 . Second, 2014-2015 seemed a turning point for many departments in the adoption of hypofractionation, and 4 out of 11 showed utilisation rates near $30 \%$. Finally, by 2015 , there were still four centres using the technique less than $25 \%$ of the time, highlighting wide differences in use between centres.

\section{Qualitative assessment}

The results of the quantitative assessment can be interpreted in light of the criteria determining the use of hypofractionation in each department. We analysed criteria concerning clinical factors emerging from the interviews on the basis of specific indications and created three categories to describe the use of hypofractionation in each department as physician-dependent, attributable to most professionals (including the reference ones for breast cancer), or with higher degree of homogeneity: a unified practice (Fig. 4). 


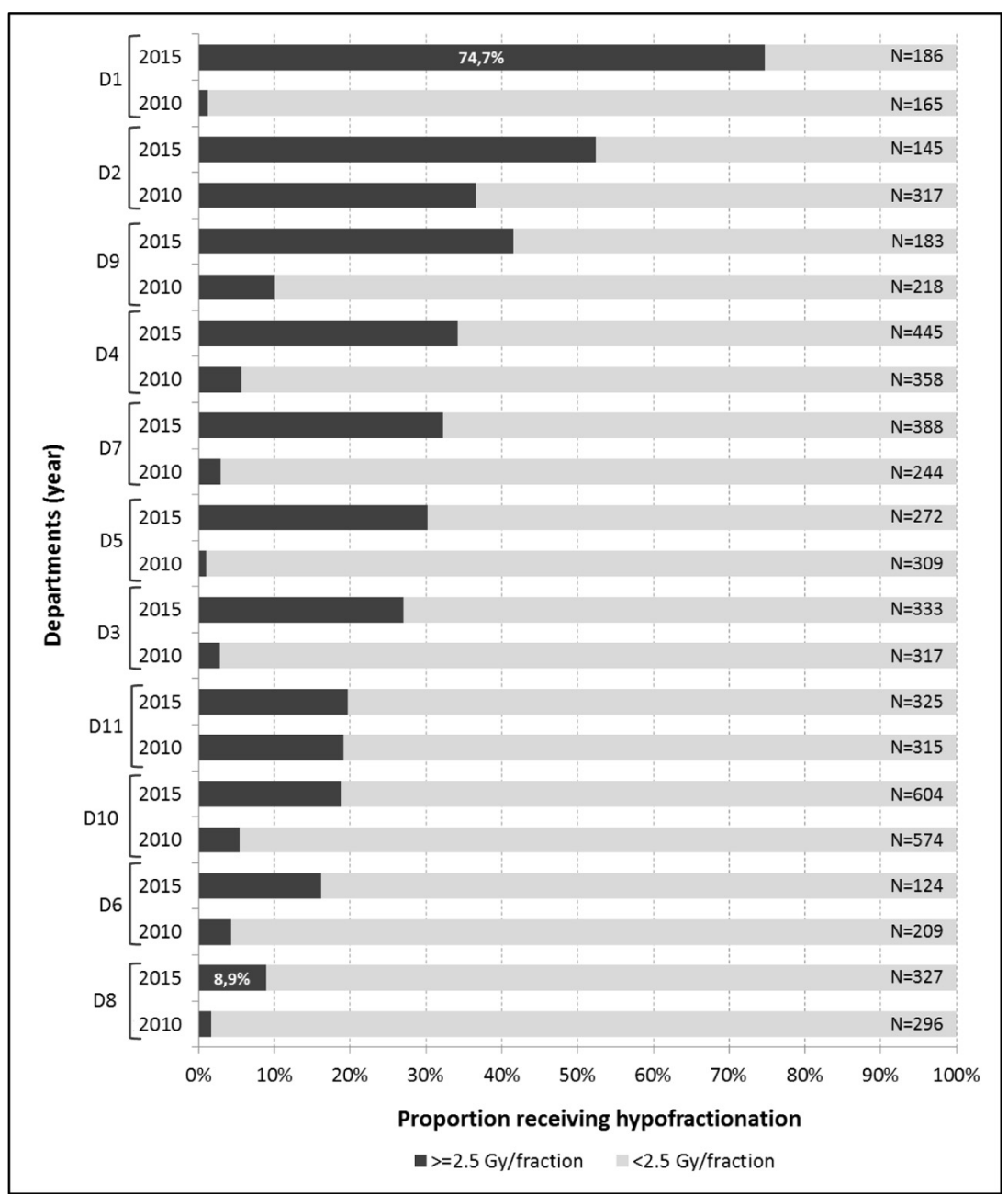

Footnote: column on the right indicates the total number of treatments delivered in the referred year.

Fig. 1. Hypofractionation use by department in 2010 and 2015.

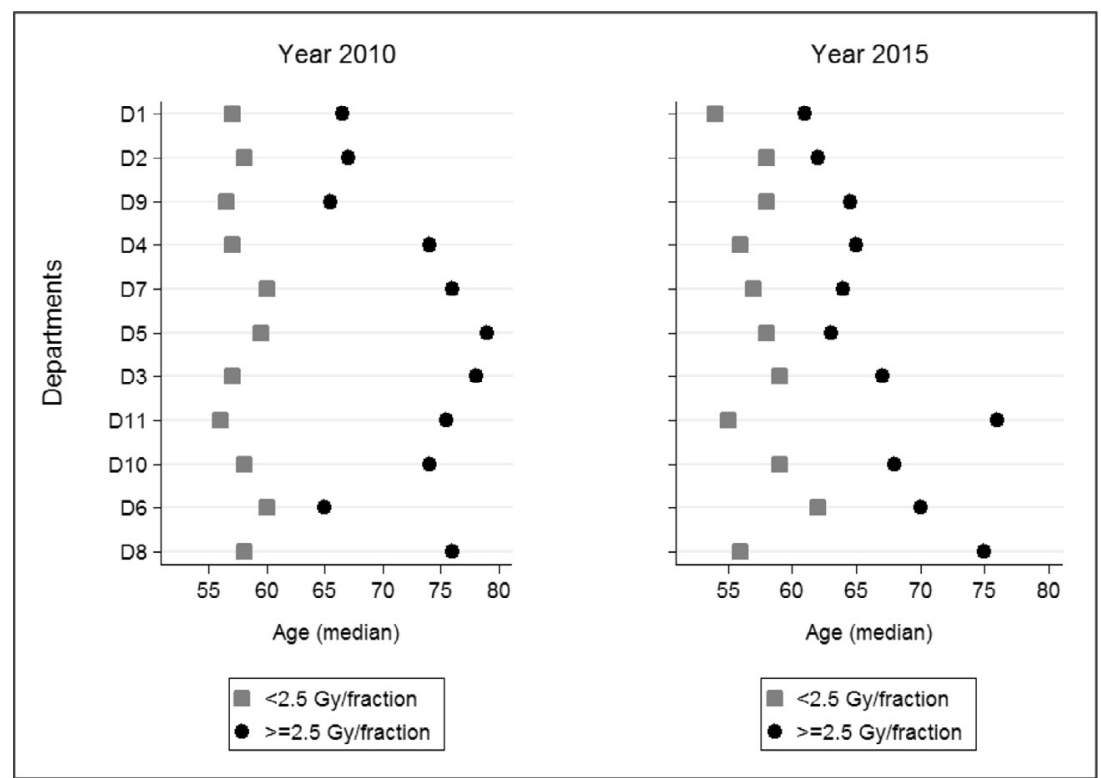

Fig. 2. Median age of patients undergoing hypofractionated radiotherapy per department in 2010 and 2015. 


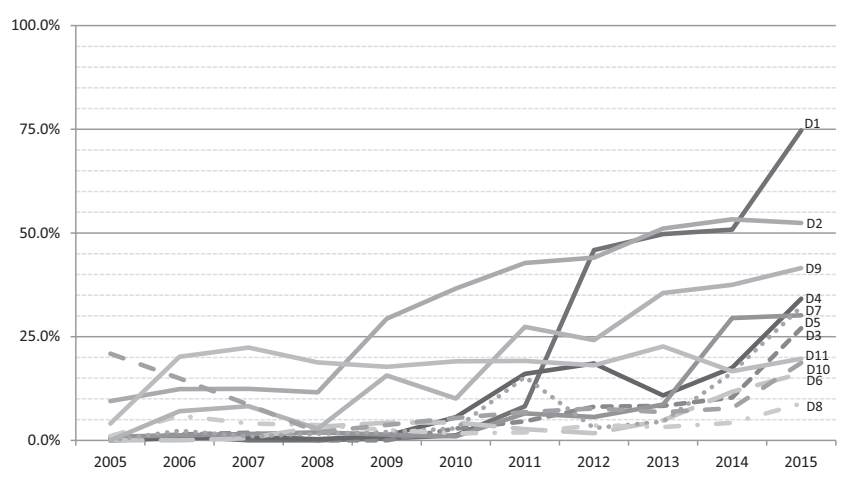

Fig. 3. Trends in use of hypofractionated whole-breast radiotherapy (HWBRT) by department (2005-2015).

The second category responds to the fact that breast cancer is rarely managed by only one or two radiation oncologists within departments due to the high caseload.

The results show the differences observed across and within clinical departments:

\section{Hypofractionation across clinical departments}

\section{"Low-risk" patients}

All interviewees pointed to the non-inferiority of hypofractionation compared to the conventional schedule, although they differed as to whether the available evidence was qualitatively consistent and sufficient to routinely adopt it. Most or all of the so-called 'low-risk' patients, that is, those whose advanced age put them at lower risk for the late side effects associated with larger doses of radiation therapy due to shorter life expectancy, were hypofractionated in 9 out of 11 departments (Fig. 4). From a chronological perspective, this is consistent with the two-wave adoption process across providers: from older to younger patients while extending indications. However, some radiation oncologists restricted hypofractionated radiotherapy to very old patients with transportation difficulties until as late as 2014, which is consistent with the median age analysis (Fig. 2). In general, the potential correlation between hypofractionation and long-term toxicity, especially cardiotoxicity, was a major cross-cutting concern among clinicians. Indeed, two clinicians openly explained reverting to the conventional schedule when their patients experienced toxicities after the course of the treatment.

\section{Chemotherapy and trastuzumab (TTZ)}

One set of indications with important differences between providers was for patients receiving chemotherapy with or without TTZ. For most professionals, combination hypofractionation plus chemotherapy was a controversial subject regardless of patients' age, while a few accepted the schedule of 40-42 Gy delivered in 15-16 fractions. However, within this favourable group, some considered primary systemic treatment "an indicator of intermediate toxicity" and only hypofractionated patients with adjuvant chemotherapy. Others included only patients receiving neoadjuvant treatment, arguing that "a longer time-lapse between chemotherapy and radiotherapy decreases the possibility of interaction and chronic toxicity".

Interestingly, many professionals stressed changing their opinion over time on the potential interaction between chemotherapy and hypofractionation. Reasons included RCT findings on cardiotoxicity during follow-up, the significant positioning of ASTRO, and particularly, their observance of equivalent or lower shortand medium-term toxicity in their patients compared to the conventional scheme.

\section{Left-sided breast and RNI irradiation}

Other important points involved hypofractionation of the 'leftsided breast' and of RNI. The former case clearly showed that professionals do not adopt hypofractionation unless they consider it a comprehensive alternative in whole-breast radiation, even with theoretical modelling showing similar local control when avoiding irradiation in part of the breast. Clinicians saw the following situations as problematic: the concomitant application of other radiotherapy techniques to avoid heart irradiation and/or the need to avoid late side effects in women with other risk factors such as hypertension or cholesterol.

In the case of RNI, almost all interviewees referred to the lack of sufficient evidence to routinely adopt it, while a few pointed out that no studies have disproven increased normal tissue effects of the brachial plexus, and there are consistent reports of successful case-by-case hypofractionation.

\section{Large/pendulous breasts, grade 3, breast cancer sub-types and chest} wall

A third set of indications gathered situations like "large/pendulous breasts", "grade 3", "breast cancer sub-types" or "chest wall". These patients were mainly hypofractionated in centres where this was the prevailing scheme. Elsewhere, hypofractionating these patients added another layer of complexity for clinicians on top of other "controversial" situations (e.g. chemotherapy, carcinoma in-situ) or techniques, for instance, using respiratory-gated radiation or decubitus prone position in the event of a large breast in order to better homogenise the dose.

\section{Boost irradiation}

Hypofractionation of the tumour bed boost also deserves a mention. Some professionals lamented the "original sin" of pivotal

Table 1

Hypofractionated radiotherapy treatments for the period 2005-2015 and adjusted OR for the annual trend of utilisation

\begin{tabular}{|c|c|c|c|c|c|c|}
\hline \multirow[t]{2}{*}{ Year } & \multicolumn{2}{|c|}{$<2.5 \mathrm{~Gy} /$ fraction } & \multicolumn{2}{|c|}{$\geq 2.5 \mathrm{~Gy} /$ fraction } & \multirow[t]{2}{*}{ OR } & \multirow[t]{2}{*}{$95 \% \mathrm{CI}$} \\
\hline & $\mathrm{N}$ & $\%$ & $\mathrm{~N}$ & $\%$ & & \\
\hline 2005 & 2019 & 93.3 & 146 & 6.7 & 1 & \\
\hline 2006 & 2673 & 92.8 & 207 & 7.2 & 1.09 & $0.86-1.38$ \\
\hline 2008 & 2913 & 95.9 & 123 & 4.1 & 0.63 & $0.49-0.82$ \\
\hline 2009 & 3064 & 93.1 & 228 & 6.9 & 1.12 & $0.89-1.41$ \\
\hline 2010 & 3038 & 91.5 & 284 & 8.5 & $1.52^{*}$ & $1.22-1.90$ \\
\hline 2011 & 3106 & 86.7 & 478 & 13.3 & $2.85^{*}$ & $2.31-3.51$ \\
\hline 2014 & 2803 & 80.7 & 672 & 19.3 & $5.15^{*}$ & $4.20-6.33$ \\
\hline 2015 & 2366 & 71.0 & 966 & 29.0 & $9.91^{*}$ & $8.09-12.13$ \\
\hline TOTAL & 30537 & 87.6 & 4322 & 12.4 & & \\
\hline
\end{tabular}

Footnote: OR: odds ratio. CI 95\%: confidence interval. Logistic regression model adjusted for patient age and department. ${ }^{*} p<0.001$ compared to reference year (2005). 


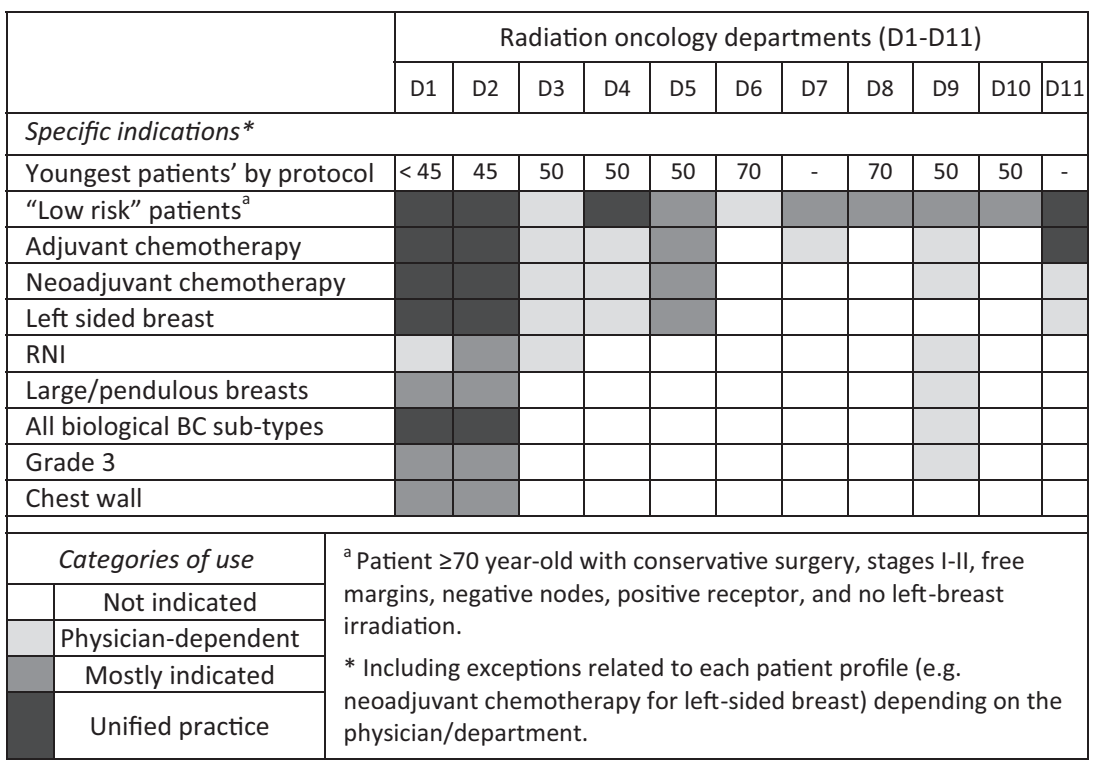

Fig. 4. Categories of use of breast cancer hypofractionation by specific indication at clinical department level (November 2015).

trials in using conventional fractionation for the boost, or in excluding hypofractionation, which paved the way for subsequent misinterpretation and variability at health system level.

\section{Hypofractionation within clinical departments}

\section{Clinical factors}

An inconsistent use of hypofractionation was shown in four departments (3, 4, 6 and 9) in which the category "physician-dep endent" matched most of the indications used (Fig. 4). Likewise, departments treating fewer patients (6,8 and 10) also failed to unify practice. Some department heads drew a contrast with prostate cancer, approached in a more homogeneous way since the beginning. The use of hypofractionation with patients who received chemotherapy was the main source of intra-department variability; this was clear in 5 of the 11 departments (Fig. 4).

\section{Clinical management factors}

Management and cultural factors also influenced the adoption of hypofractionation. Besides the (widely acknowledged) inertia existing in clinical departments, many professionals saw hypofractionation as "something to believe". Respondents pointed to four factors:

(1) The role played by the department heads: Some heads operated under the assumption that clinical practice should be homogenous within a single department, and they deliberately stimulated innovative behaviours, while others decentralised these management decisions to specialists. Several respondents in low-hypofractionating departments associated clinical excellence and traditional approaches with statements such as, "We have good clinical outcomes compared to international experiences". Physicians who had worked in other centres were privileged witnesses to the impact of organisational culture on physicians' use of varying radiation schemes.

(2) The place of hypofractionation in the clinical protocol: While most departments considered hypofractionation to be one "possibility of two alternatives" in accordance with their protocol, one gave it "priority over the conventional schedule". A third type of protocol restricted its use to specific cases "up to [the protocol's] comprehensive update", that is, once every 1-2 years, making the timing of this revision a barrier for introducing new schemes. Notably, some departments set a minimum patient age of 50 for hypofractionation (Fig. 2); however, the median age analysis showed that it was delivered (in some cases almost exclusively) to patients over 70 (Fig. 1).

(3) Ties between radiation specialists: Respondents mostly appraised professional ties as a failed driver in adopting hypofractionation. Young physicians stressed the need to improve communication and overcome the current silobased departmental model.

(4) Change of pattern in machine use: Changes in machine usage due to routine hypofractionation are considered relevant, though it is unclear whether this would increase or decrease use.

\section{Discussion}

This study assessed the patterns of use and adoption of HWBRT for the conservative treatment of breast cancer in the public healthcare sector of Catalonia (Spain) from 2005 to 2015. This is an example of diffusion of change in practice where no specific indications for this treatment have been established by the regional clinical guideline. Although use of HWBRT is increasing region-wide, important variations remain in the rate of adoption, which ranged from $8.9 \%$ to $74.7 \%$ in 2015 . A population-based study in New South Wales (Australia) [11] showed similar findings, reporting an average rate of utilisation of $35 \%$ (compared to $29 \%$ for 2015 in our study) and variability in the rates of adoption among departments, ranging from $6 \%$ to $92 \%$ for $2007-2012$. In our study, the time-frame 2014-2015 seemed to be a turning point towards the mainstream adoption of hypofractionation, although only 2 out of 11 departments used it more than $50 \%$ of the time, and 4 others basically limited hypofractionation to patients over 70 , highlighting how far this regimen is from being considered a routine practice. The qualitative analysis identified barriers to its uptake for particular indications and illustrated how frequently its use depends on physicians' prerogative.

The integration of both quantitative and qualitative strands of research allowed us to identify three patterns for adopting hypofractionation among all radiotherapy departments: 
accelerated adoption, involving the substitution of conventional treatment (dept. 1, 2 and 9); progressive adoption, showing the coexistence between radiation schemes (dept. 3, 4, 5 and 7); and experimental adoption, based on case-by-case hypofractionation, mainly in elderly ( $>70$ years) patients (dept., 6, 8, 10 and 11 ). These results suggest that understanding the Diffusion of Innovations theory may shed light on how hypofractionation is adopted across the health systems [29]. For instance, this theory suggests that the passage of time would interact with external and internal influences (e.g. perceived efficiency gained in relation to the classical scheme, specific recommendations at international level, less acute toxicity observed, etc.) and stimulate adoption of the innovation in 2014 by the so-called 'early majority' of five departments.

To speed up the dissemination process, Chapman et al. [30] described the phased implementation of a 5-year clinical pathway for breast radiation therapy that increased the utilisation rates from $8.3 \%$ to over $75 \%$. In Catalonia, our analysis revealed that while indications were not mutually exclusive, chemotherapy with or without TTZ worked as a stopcock within them, especially for women under 70 . The hypofractionation of patients receiving chemotherapy and, similarly, the occurrence of long-term toxicity, was the main point of disagreement among clinicians and source of variability in clinical practice at intra- and inter-department levels. As half of all breast cancer patients fall into these categories, their inclusion in a hypofractionated regimen is a decisive determinant of its overall use. The DBCG HYPO randomized clinical trial provides new evidence on this issue by including $36 \%$ of patients with chemotherapy, with no associated increase in toxicity [31].

Nevertheless, the analysis of clinicians' rationale highlighted the importance of interpreting hypofractionation use in the context of the specific indications rather than from a broader perspective. Specialists' views sometimes reflected discrepancies already discussed in the literature, such as with RNI or tumour-grade sensitivity $[13,14,32]$. Strikingly, in other situations clinicians shared interpretation of the evidence, for example agreeing that theoretical modelling showed similar local control on avoiding irradiation in part of the left-sided breast, but clinical practice differed anyway. There are two ways of understanding this phenomenon: first, although Delaney and colleagues indicated that newer techniques, such as deep inspiration breath-hold techniques to decrease the risk of heart damage, should diminish concerns [11], some clinicians perceived these concomitant techniques as "another layer of complexity", and they seemed to slow HWBRT's uptake. Second and more relevantly, quality of evidence is a necessary but not a sufficient condition determining clinicians' behaviour towards hypofractionation. Clinical management factors, such as the role of the department head, played a key role in explaining the adoption of this therapeutic strategy, for example in the D1 department's sudden increase in use in 2011 (Fig. 3).

The HWBRT has proven to be a controversial therapeutic strategy in its implementation, leading to different rhythms of adoption and characterised by intra- and inter-department variability. Clinicians suggested during discussions that an updated clinical guideline would contribute to homogenising clinical practice and/or addressing treatments' cost-effectiveness, thus contributing to streamlining a process of decision-making currently restricted to clinicians' criteria. Furthermore, some authors noted that there is no reason to assume that this approach is limited to modest hypofractionation (15-16-fraction regimen) [6], so changes in this regard would eventually widen the existing gap between early and late adopters.

This study has some strengths and limitations. Regarding the quality of the quantitative data, we used a specific registry covering all the radiotherapy departments in Catalonia, whose comprehensiveness is reinforced through linkage to treatment reimbursement. We also performed statistical analyses using data exclusively from the public sector (responsible for providing more than $85 \%$ of services). One limitation was that clinical factors such as patient stage or chemotherapy indication were not taken into account.

Strengths of the qualitative study were the comprehensive sample, including all department heads and reference specialists for breast cancer. As far as limitations are concerned, unlike other studies [10,33], we did not approach the topic of travel, although one hospital raised the issue due to its geographic location. Moreover, in contrast to other experiences [34-36], neither financial pressures nor waiting lists were of relevance in the analysis of the adoption of hypofractionation.

In conclusion, the clinical practice of breast cancer hypofractionation showed considerable variability due to both providerbased factors and along the course of adoption in the Catalonian NHS. These differences are rooted in clinicians' interpretation of the evidence, especially concerning potential long-term toxicity, but also in relation to context-dependent factors that significantly favoured or hindered professional trust towards this new scheme. In tackling with differences in the rate of adoption of HWBRT at the health system level, a rational, evidence-based approach should ideally converge with professional perspectives, the factors influencing the interpretation of the evidence, and the organisational context, including existing dissemination channels.

\section{Role of the funding source}

None.

\section{Conflict of Interest statement}

The authors declare no conflict of interest.

\section{Acknowledgements}

We are grateful to Marta Bonet (MD), Xavi Sanz (MD), Josep Antoni Carceller (MD), Ferran Guedea (MD), Antonio Arellano (MD), Jordi Craven-Bartle (MD), Josep Isern (MD), Albert Biete (MD), Josep M. Solé (MD), Joan Carles Julià (MD), Jordi Giralt (MD), and Jordi Vayreda (MD), for their special contribution to the work. This work was supported by the Carlos III Institute of Health (Instituto de Salud Carlos III/ISCIII) through the Cancer Research Network (co-funded by FEDER funds / European Regional Development Funds, ERDF) (RD 12/0036/0053); and the Agència de Gestió d'Ajuts Universitaris i de Recerca (AGAUR, 2014SGR0635). These institutions played no role in the design of the study, collection, analysis and interpretation of data, and in writing the manuscript. Further, we are grateful to Ms. Meggan Harris for her editorial support.

\section{References}

[1] Whelan T, MacKenzie R, Julian J, et al. Randomized trial of breast irradiation schedules after lumpectomy for women with lymph node-negative breast cancer. J Natl Cancer Inst 2002;94(15):1143-50.

[2] Whelan T, Pignol J, Levin M, et al. Long-term results of hypofractionated radiation therapy for breast cancer. NEJM 2010;362:513-20.

[3] Bentzen SM, Agrawal RK, Aird EG, et al. The UK Standardisation of Breast Radiotherapy (START) Trial B of radiotherapy hypofractionation for treatment of early breast cancer: a randomised trial. Lancet 2008;371:1098-107.

[4] Haviland JS, Owen JR, Dewar JA, et al. The UK Standardisation of Breast Radiotherapy (START) trials of radiotherapy hypofractionation for treatment of early breast cancer: 10-year follow-up results of two randomised controlled trials. Lancet Oncol 2013;14:1086-94.

[5] Montero A, Sanz X, Hernanz R, et al. Accelerated hypofractionated breast radiotherapy: FAQs (frequently asked questions) and facts. Breast 2014;23:299-309.

[6] Yarnold J, Haviland J. Pushing the limits of hypofractionation for adjuvant whole breast radiotherapy. Breast 2010;19:176-9. 
[7] Arenas M, Sabater S, Gascón M, et al. Quality assurance in radiotherapy: analysis of the causes of not starting or early radiotherapy withdrawal. Radiat Oncol 2014;9:260

[8] Haffty BG, Buchholz TA. Hypofractionated breast radiation: preferred standard of care? Lancet Oncol 2013;14:1032-4.

[9] Koulis TA, Phan T, Olivotto IA. Hypofractionated whole breast radiotherapy: current perspectives. Breast Cancer (Dove Med Press) 2015;27:363-70.

[10] Wang EH, Mougalian SS, Soulos PR, et al. Adoption of hypofractionated wholebreast irradiation for early-stage breast cancer: a National Cancer Data Base analysis. Int J Radiat Oncol Biol Phys 2014;90:993-1000.

[11] Delaney GP, Gandhidasan S, Walton R, Terlich F, Baker D, Currow D. The pattern of use of hypofractionated radiation therapy for early-stage breas cancer in New South Wales, Australia, 2008 to 2012. Int J Radiat Oncol Biol Phys 2016;96:266-72.

[12] Jagsi R, Falchook AD, Hendrix LH, Curry H, Chen RC. Adoption of hypofractionated radiation therapy for breast cancer after publication of randomized trials. Int J Radiat Oncol Biol Phys 2014;90:1001-9.

[13] Whelan TJ, Pignol JP, Levine MN, et al. Long-term results of hypofractionated radiation therapy for breast cancer. N Engl J Med 2010;362:513-20.

[14] Haviland JS, Yarnold JR, Bentzen SM. Hypofractionated radiotherapy for breast cancer. N Engl J Med 2010;362:1843.

[15] Whitfield GA, Horan G, Irwin MS, et al. Incidence of severe capsular contracture following implant-based immediate breast reconstruction with or without postoperative chest wall radiotherapy using 40 Gy in 15 fractions. Radiother Oncol 2009;90:141-7.

[16] Arenas M, Montero Á, de Las Peñas MD, Algara M. The position and current status of radiation therapy after primary systemic therapy in breast cancer: a national survey-based expert consensus statement. Clin Transl Oncol 2016;18:582-91.

[17] Budach W, Bölke E, Matuschek C. Hypofractionated radiotherapy as adjuvant treatment in early breast cancer. A review and meta-analysis of randomized controlled trials. Breast Care 2015;10:240-5.

[18] Manchon P, Borràs JM, Ferro T, Espinàs JA, Breast Cancer OncoGuia Group. Breast Cancer OncoGuia. Clin Transl Oncol 2010;12(2):113-38.

[19] Creswell JW, Plano Clark VL. Designing and conducting mixed methods research. USA: Sage Publications; 2011.

[20] Morse JM, Niehaus L. Mixed methods design: principles and procedures. Walnut Creek, CA: Left Coast Press; 2009.
[21] Teddlie C, Tashakkori A. Foundations of mixed methods research. Thousand Oaks, CA: Sage; 2009

[22] Bryman A. Integrating quantitative and qualitative research: how is it done? Qualitative research 2006;6:97-113.

[23] Denzin NK, Lincoln YS. The SAGE handbook of qualitative research. Thousand Oaks: Sage Publications; 2005.

[24] Sofaer S. Qualitative research methods. Int J Qual Health Care 2002;14:329e36.

[25] Dahlgren L, Emmelin M, Winkvist A. Qualitative methodology for international public health. Umea, Sweden: Umea University; 2004.

[26] Guest G, Bunce A, Johnson L. How many interviews are enough? An experiment with data saturation and variability. Field Meth 2006;18:59-82.

[27] Strauss A, Corbin J. Basics of qualitative research. Techniques and procedures for developing Grounded Theory. Thousand Oaks, US: Sage Publications. 1998.

[28] Muhr T. ATLAS.ti v6.2 for Windows. Berlin: Scientific Software Development. 2011.

[29] Rogers EM. Diffusion of innovations. New York: 5th ed., The Free Press of Glencoe; 2003.

[30] Chapman BV, Rajagopalan MS, Heron DE, Flickinger JC, Beriwal S. Clinical pathways: a catalyst for the adoption of hypofractionation for early-stage breast cancer. Int J Radiat Oncol Biol Phys 2015;93:854-61.

[31] Offersen BV, Jacobsen EH, Nielsen MH, et al. Hypo- vs normofractionated radiation of early breast cancer in the randomised DBCG HYPO trial. Radiother Oncol 2016:119:S64-5.

[32] Badiyan SN, Shah C, Arthur D, et al. Hypofractionated regional nodal irradiation for breast cancer: examining the data and potential for future studies. Radiother Oncol 2014;110:39-44.

[33] Pagano E, Di Cuonzo D, Bona C, et al. Accessibility as a major determinant of radiotherapy underutilization: a population based study. Health Policy 2007;80:483-91.

[34] Bekelman JE, Sylwestrzak G, Barron J, et al. Uptake and costs of hypofractionatedvs conventional whole breast irradiation after breast conserving surgery in the United States, 2008-2013. JAMA 2014;312:2542-50.

[35] Lievens Y. Hypofractionated breast radiotherapy: financial and economic consequences. Breast 2010;19:192-7.

[36] Dwyer P, Hickey B, Burmeister E, et al. Hypofractionated whole-breast radiotherapy: impact on departmental waiting times and cost. J Med Imaging Radiat Oncol 2010;54:229-34. 